\title{
la cerámica de construcción en Norteamérica
}

\author{
F. ARREDONDO VERDÚ \\ $691.42(13)=60^{\text {Ingeniero de Caminos }}$
}

Teniendo en cuenta que sólo hemos visitado una fábrica de ladrillos, la Davidson Brick Company, y los Iaboratorios de la Structural Clay Products Research Foundation, es imposible exponer características detalladas de la fabricación cerámica en los Estados Unidos.

No obstante, de las conversaciones mantenidas con los técnicos de estas dos entidades, así como con los de las fábricas de bloques de mortero, fuertes competidoras de los ladrillos, hemos podido fijar las siguientes ideas sobre dicha industria.

Toda la industria norteamericana de la construcción está basada en tres principios fundamentales:

1.9) Economía de mano de obra.

2.9) Corta duración de los edificios.

$3 .^{\circ}$ Aumento incesante de la producción por hombre-día.

La industria cerámica no habia de verse fuera de este cuadro general y tiene, por tanto, que adaptarse a esta modalidad industrial.

Las consecuencias lógicas de estos principios fundamentales son la mecanización, la producción de grandes series y un desvio de los materiales tradicionales durables para caminar hacia otros que, por no exigírseles durabilidad, pueden ser más económicos.

Debido, seguramente, a esta última circunstancia, ha desaparecido casi totalmente la teja como material de cubrición.

Es evidente que el ladrillo es un material que se presta admirablemente a la gran serie; y no sólo que se presto, sino que es obligada su producción en tal forma para que resulte eminenfemente barato. Llevando al límite esta consideración, Ilegan en Estados Unidos al extremo de que cada fábrico no suele producir más que un tipo de ladriIlo. Ampliando este criterio de cada fábrica a todo el país, resulta que casi el único ladrillo fabricado es el macizo ordinario o de paramento que, por otra parte, es el que más exige el mercado, dadas las formas de construcción en práctica, con materiales ligeros de fácil colocación en obra y relativamente poco durables.

La mecanización abundante aumenta, como es natural, la productividad y resulta económico cuando la energía es barata, como ocurre en este caso.

Como la principal utilización de los ladrillos es como material de cerramiento en grandes edificios, las fábricas de ladrillos se sitúan en las cercanías de las ciudades y generalmente a pie de cantera.

En cualquier punto de los Estados Unidos se dispone, en forma abundante y barata, de electricidad $y$ de combustibles sólidos, líquidos y gaseosos. No hay, pues, problema de energía para aumentar cada vez más la mecanización, ya que, mientras en España el kilovatio-hora eléctrico, por ejemplo, cuesta de $1 / 15$ a $1 / 20$ de la hora de peón, en Norteamérica no cuesta más que 1/100. 
Se dispone, en general, de material y maquinaria ampliamente dimensionada, con lo cual el rendimiento de la mano de obra es magnífico, pero el de la energía es mediano.

La explotación de canteras se realiza con moderna maquinaria para excavacione's y se consigue un alto rendimiento.

En el moldeo predomina la máquina de vacío con boquillas muy estudiadas por vía experimental. Es sorprendente la capacidad de los motores de estas máquinas, pues oscila entre los 150 y $300 \mathrm{CV}$, lo que supone una potencia absorbida de unos $10 \mathrm{CV} / \mathrm{Tm} /$ hora.

El secadero más uțilizado es el secaderotúnel, verificándose el secado, generalmente, con aire puro y no con los humos conteniendo los productos de la combustión.

El hecho de que cada fábrica produzca un número muy limitado de tipos de ladrillos, generalmente nada más que uno, es una circunstancia favorable a la utilización del horno-túnel, que cada día se utiliza más, en detrimento de los hornos intermitentes usados anteriormente. El consumo por término medio, es de unos $50 \mathrm{~kg}$ de carbón de 7.000 calorías $/ \mathrm{kg}$ por tonelada de productos cocidos. Si se emplean otros combustibles, el consumo es equivalente. El gasto de energía es de unos $5 \mathrm{CV} / \mathrm{Tm} / \mathrm{dí}$.

Creemos interesante resaltar la importancia que se da al control de la fabricación, estando, todos los elementos que intervienen en el proceso, dotados de numerosos indicadores o registradores, de forma que, en cualquier momento, puede saberse en que condiciones se encuentra el producto en curso de fabricación. Esto encarece muy poco la instalación, y se trata, desde luego, de una inversión muy rentable, ya que se traduce en una mejora de la calidad.

Refiriéndonos concretamente a la fábrica visitada, podemos decir que utilizan prensas de vacío y secaderos-túnel en los que el $29 \%$ de agua de moldeo se reduce a 1 ó $2 \%$; con un buen control, las pérdidas por roturas en esta fase de la producción ascientden tan sólo al 3 ó $4 \%$. En los secaderos, con 54 vías de $35,6 \mathrm{~m}$, permanecen los ladrillos 2 días. El aire caliente producido en hogares auxiliares entra en el secadero en los 10 primeros metros.
La cocción se realiza en hornos de hormiguero o similares, alimentados con gas natural. La temperatura de cocción oscila entre los $1.065^{\circ} \mathrm{C}$ y $1.150^{\circ} \mathrm{C}$.

Con este sistema de cocción se obtiene un 3 ó $4 \%$ de material no aprovechable, el cual suele utilizarse en formar la pared exterior del horno en la cocción siguiente.

No fabrican más que material macizo de las siguientes dimensiones:

$$
\begin{aligned}
& 28,9 \times 8,5 \times 7,6 \mathrm{~cm} \\
& 25,4 \times 8,25 \times 8,25 \mathrm{~cm} \\
& 20,9 \times 6,3 \times 9,8 \mathrm{~cm}
\end{aligned}
$$

La superficie externa de los ladrillos puede quedar lisa, como sale de la máquina, o con unas ranuras irregulares que dan movimiento a la fachada si se utilizan a cara vista o proporcionan un excelente agarre para los enlucidos si se efectúan éstos.

\begin{tabular}{|c|c|c|c|}
\hline \multicolumn{2}{|c|}{$\begin{array}{l}\text { Mínime resistencia } \\
\text { a compresión }\end{array}$} & \multicolumn{2}{|c|}{$\begin{array}{c}\text { Maxima absorción } \\
\text { en aguo hiviendo } \\
\text { durante } 5 \text { horas }\end{array}$} \\
\hline $\begin{array}{l}\text { Promedio } \\
\text { des } 5 \\
\text { probetas } \\
\mathrm{kg}^{\prime} / \mathrm{cm}^{2}\end{array}$ & $\begin{array}{l}\text { Individual } \\
\mathrm{Kg} / \mathrm{cm}^{2}\end{array}$ & $\begin{array}{c}\text { Promedia } \\
\text { de } 5 \\
\text { probetas } \\
\% \\
\%\end{array}$ & $\begin{array}{c}\text { Individual } \\
\%\end{array}$ \\
\hline 175 & 155 & 22,0 & 25,0 \\
\hline 105 & 88 & $\begin{array}{l}\text { Nose se } \\
\text { litimita }\end{array}$ & $\begin{array}{l}\text { No se } \\
\text { Iimitat }\end{array}$ \\
\hline
\end{tabular}

Las características del material fabricado por esta fábrica son las siguientes:

Si bien es verdad que la tonelada de ladrillos cuesta poco más o menos que la tonelada de otros materiales en competencia con aquéllos, no sucede lo mismo con el precio de la obra de fábrica realizada con unos u otros materiales. En este aspecto, el ladrillo está en franca desventaja.

Para luchar con esta competencia dura que presentan ofros productos, principalmente los bloques de cemento, se creó, en el año 1950, la Structural Clay Products Research Foundation, patrocinada y subvencionada por fabricantes norteamericanos y canadienses que, en un principio, eran 110 de los primeros y 28 de los segundos. 
Actualmente se ha ampliado la participación de fabricantes, que contribuyen con el $0,5 \%$ de sus cifras de ventas, manejando la Fundación un presupuesto anual de 500.000 dólares.

En varias direcciones ha trabajado esta Fundación.

Una de ellas ha sido en el estudio de un nuevo tipo de ladrillo que aumenta la productividad por sus caracteristicas.

Los muros tradicionales de las casas americanas son de 8" $(20 \mathrm{~cm})$, pero todas las partes interesadas están de acuerdo en que este espesor es excesivo para la vivienda unifamiliar de un solo piso que tanto abunda en aquel pais. Los más modernos Códigos de Edificación, tanto federales como locales, permiten en estas casas el uso de muras de cargo de $6^{\prime \prime}(15 \mathrm{~cm})$ de grueso.
Entonces, la S. C. P. R. F. estudió un nuevo tipo de ladrillo para adaptar las ventajas antiguas de lo fábrica de ladrillo a las tendencias constructivas modernas, con vistas a abarałar la construcción de viviendas.

Después de unos estudios exhaustivos de tiempos y movimientos, se llegó al ladrillo que tiene por dimensiones nominales $2 z / 9 x$ $6 \times 12$ pulgadas $(6,7 \times 15,2 \times 30,4 \mathrm{~cm})$. Como se construye con juntas de $1 / 2^{\prime \prime}(1,25 \mathrm{~cm})$, las dimensiones resultantes son $2 \% 10 \times 5 \frac{1}{2} \times$ $11 \frac{1 / x}{\text { pulgadas }}(5,5 \times 14 \times 29,2 \mathrm{~cm})$ (Figura 1$)$.

El peso aproximado de esta pieza es de 3.700 gramos.

Tiene diez perforaciones cilíndricas, de 1 " $/ 8$ " de diámetro $(3,5 \mathrm{~cm})$. Estas perforaciones permiten cogerlo fácilmente con una mano y al mismo tiempo favorece la adherencia por el mortero introducido en estas perforaciones.

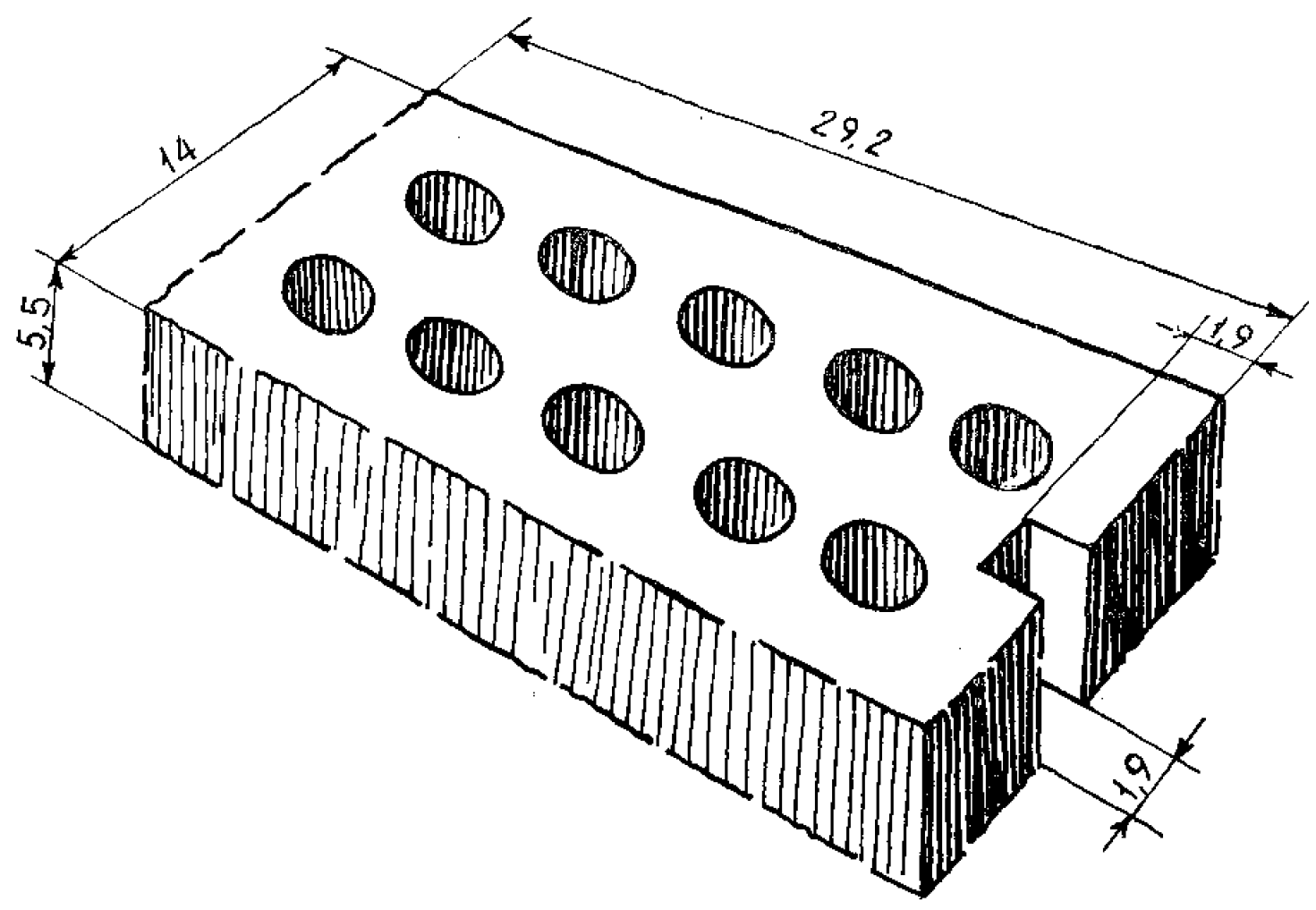

Fig. 1 
Se le ha dotado además de una ranura en una de las caras menores para facilitar la colocación de ventanas y puertas.

Con estas unidades se construyen las fábricas de medio pie que constituyen los muros de carga de una vivienda.

Se requieren 450 ladrillos para la construcción de 100 pies $\left(9,3 \mathrm{~m}^{2}\right)$ de muro de un pie, lo cual puede ser realizado sin esfuerzo por un hombre en un día. Comprobaciones recientes indican que esta cifra es baja. La productividad actual es mayor; generalmente un buen operario puede colocar, sin fatiga, de 500 a 600 ladrillos por jornada de ocho horas, to cual significa 110 a 130 pies $^{2}$ (10,2 a $12 \mathrm{~cm}^{2}$ ).

Esta variación de la dimensión del ladrillo respecto a las tradicionalmente empleadas, ha hecho aumentar la producción de metros cuadrados de muro por obrero y día casi en un $100 \%$. Por esta razón, la curva de ventas actuales es fuertemente creciente.

La materia prima para la fabricación de estos ladrillos es la misma que para los demás y el proceso de fabricación no varia lo más mínimo.
Actualmente se está tratando de la fabricación de este modelo de ladrillo, pero en tipo ligero, mediante la dilatación previa de la arcilla a $1.540^{\circ} \mathrm{C}$. De los estudios realizados hasta la fecha resulta que la relación de densidades es de 1,7 a 2,4 , en tanto que la relación de resistencias es de 4,5 a 7.

Teniendo siempre como objetivo el ahorro de mano de obra de peones y albarilles se han estudiado diversos dispositivos que tienden a este fin.

Con objeto de eliminar peones se ho estudiado un tipo de empaquetado que permite colocar fácilmente, y de forma económica, bloques de 62 ladrillos al alcance de la mano del albañill, subiéndolos al andamio mediante un carro elevador corriente.

Si bien es verdad que esto ahorra mano de obra en el tajo, la aumenta en fábrica. Para evitar este aumento, se ha diseñado una máquina empaquetadora que realiza el empaquetado de los 62 ladrillos en la forma indicada en la figura 2.

Estos bloques van atados con cuatro flejes de acero.
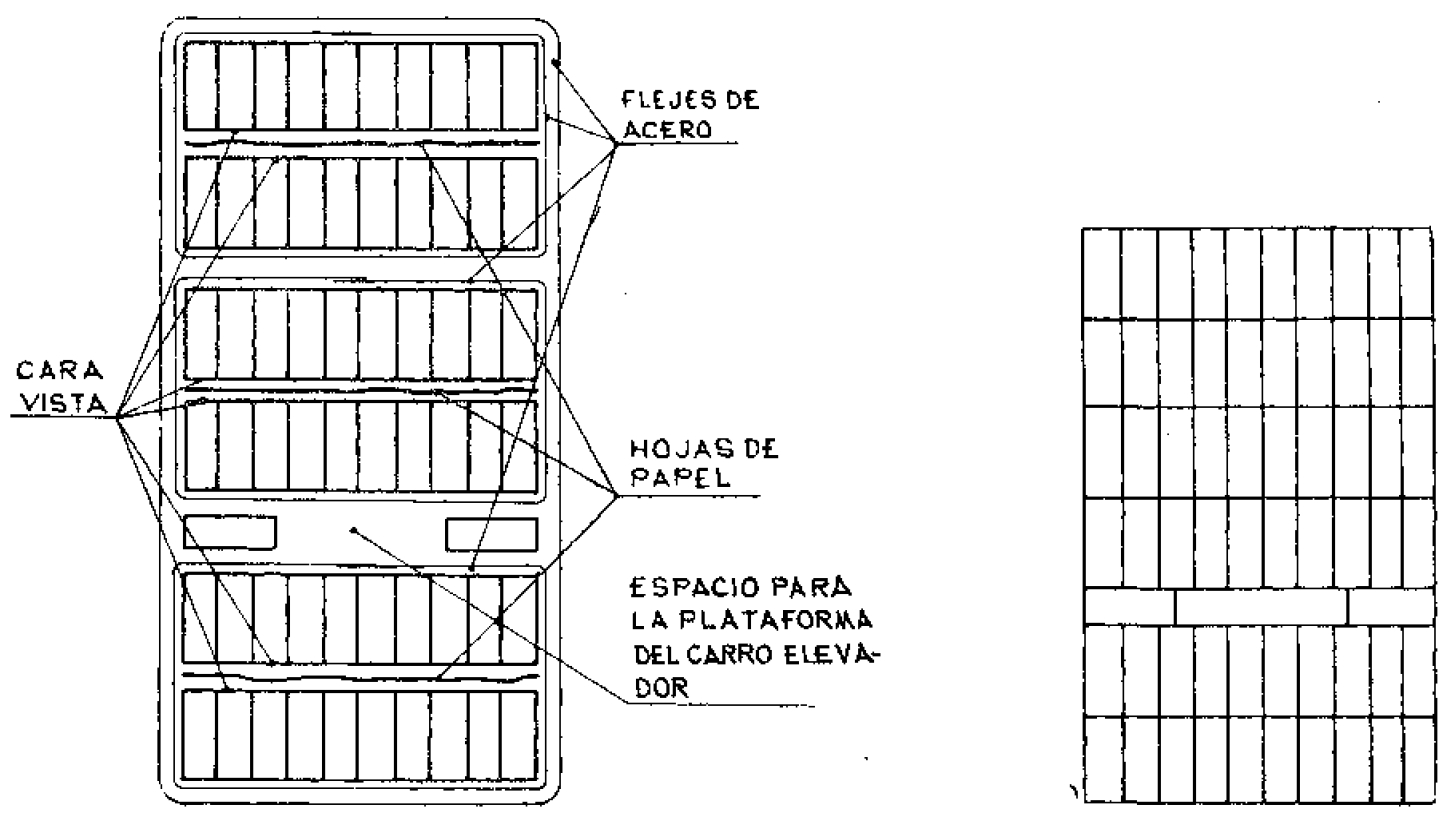

Fig. 2 
No creemos que esto sea utilizable en España en manera alguna.

Esta Fundación ha diseñado también unos andamiajes deslizantes, los cuales permiten situar al albañil en una posición cómoda respecto al muro y respecto a los materiales que ha de emplear para su construeción.

Para facilitar la construcción dispone también el operario de un elemento similar a nuestras miras con un complicado sistema de herrajes metálicos para su fijación.

Estimamos que todos estos sistemas de aumentar la productividad no susponen nada nuevo. Tal vez algún dispositivo determinado sea realmente nuevo e ingenioso. Pero en su conjunto son un sistema más de los que existen por el mundo e, incluso, en España, donde, en este sentido, ha trabajado intensamente durante varios años el Instifuto Téenico de la Construeción y del Cemento consiguiendo resultados excelentes.

Se han realizado también estudios sobre forrado exterior de casas de madera mediante plaquetas cerámicas sujetas por grapas metálicas y también paneles de muro prefabricados, eventualmente armados, con cara exterior de ladrillo y terminado interior de yeso, terracota o azulejo. La longitud máxima de estos paneles es de unos 3 metros.

Independientemente de estas cuestiones, la Fundación lleva a cabo investigaciones y ensayos sobre materiales cerámicos. Asi pues, ha realizado una serie de ensayos para asegurarse de la calidad y definir las características del ladrillo citado anteriormente. Los ensayos de compresión, flexión, distorsión e impacto se han efectuado sobre paneles de muros construídos con estos ladrillos y han demostrado cumplir ampliamente las normas vigentes en aquél país.
También han llevado a cabo ensoyos de resistencia al fuego y al chorro de agua subsiguiente, llegándose a la conclusión de que el tiempo de resistencia al fuego es de 2 horas 32 minutos y que resiste suficientemente el impacto y erosión de un chorro con una presión de $2,1 \mathrm{~kg} / \mathrm{cm}^{2}$ actuando durante dos minutos y medio.

Otro campo de estudio, también abordado por este Organismo, ha sido el de las eflorescencias. El estudio ha sido amplio, con objeto de identificar la naturaleza de las eflorescencias, averiguar el mecanismo de su formación y los medios para evitarlas.

Se ha llegado a la conclusión de que las eflorescencias, en los productos ensayados, son sales de calcio y magnesio. En particular los sulfatos cálcico y magnésico son impurezas que producen fuertes eflorescencias $y$ desgraciadamente son encontrados con frecuencia en las materias primas.

Se ha comprobado que la velocidad de secado no afectó a la formación de efiorescencias, pero el secado rápido a alta temperatura produce la uniforme distribución de las eflorescencias en las probetas. También se ha obtenido la convicción de que las atmósferas de anhidrido sulfuroso en el secadero o en el horno no favorecen la formación de eflorescencias, a menos que se cumplan, además, las siguientes condiciones:

a) Debe haber presentes sales de calcio o de magnesio para reaccionar con él para formar sulfatos.

b) Debe existir en la masa arcillosa un exceso de agua en cantidad de un 8 a $12 \%$ para actuar como vehículo que transporte las sales a la superficie, en concentración suficiente para formar las eflorescencias.

c) Hay que dejar pasar un cierto tiempo para que este proceso descrito se realice. 